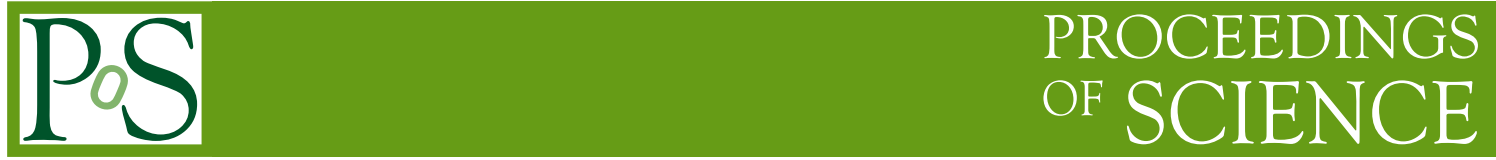

\title{
Isospin breaking and the chiral condensate
}

\section{Michael Creutz*}

Brookhaven National Laboratory

E-mail: creutz@bnl.gov

With two degenerate quarks, the chiral condensate exhibits a jump as the quark masses pass through zero. I discuss how this single transition splits into two Ising like transitions when the quarks are made non-degenerate. The order parameter is the expectation of the neutral pion field. The transitions represent long distance coherent phenomena occuring without the Dirac operator having vanishingly small eigenvalues.

XXIIIrd International Symposium on Lattice Field Theory

25-30 July 2005

Trinity College, Dublin, Ireland

\footnotetext{
* Speaker.
} 


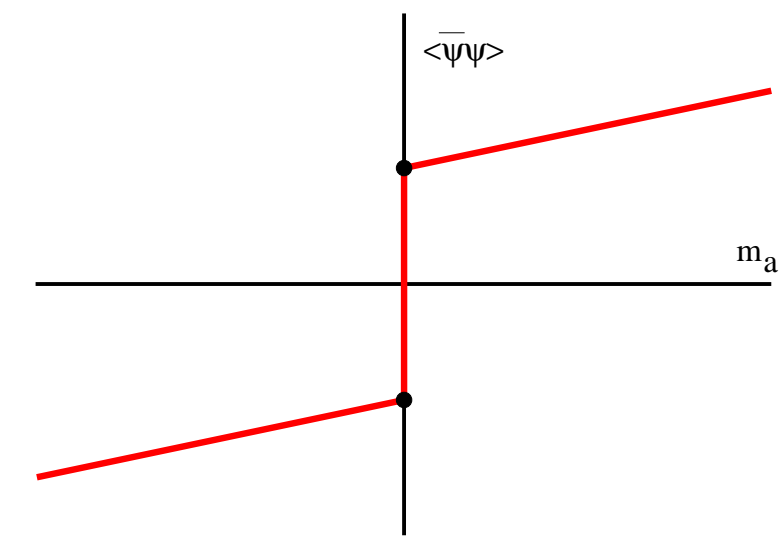

Figure 1: A sketch of how the chiral condensate jumps in sign as the quark mass passes through zero for two flavors of degenerate quark.

Spontaneous chiral symmetry breaking is a cornerstone of our understanding of low energy hadronic dynamics. In particular, the idea of pions as approximate Goldstone bosons is the accepted explanation of why they are so much lighter than the rho mesons, despite having the same quark content. In this picture, a condensate appears with $\bar{\psi} \psi$ maintaining an expectation value even for vanishing quark mass. The value of this condensate depends on the direction from which the masses approach the chiral limit. With two flavors, one expects a jump in the chiral condensate as the quark mass passes through zero. This behavior is sketched in Fig. (1). As a flavored chiral rotation can flip the sign of the mass, the physics is the equivalent on both sides of the transition.

This represents the long established picture with two degenerate quarks. The question I address here is what happens when the quarks are not degenerate. In particular, hold the up-down quark mass difference fixed and vary the average quark mass through zero. The result is a splitting of the transition into two second order transitions, as sketched in Fig. (2). These transitions are each Ising like and the order parameter is the expectation value of the neutral pion field, $\left\langle\pi_{0}\right\rangle$. The effect is quadratic in the splitting of the quark masses, the critical value for the average quark mass $m_{c}$ being proportional to $\left(m_{d}-m_{u}\right)^{2}$. Since the neutral pion is odd under CP, this symmetry is spontaneously broken in the intermediate phase. Finally I note that these transitions have nothing to do with the point where the up quark mass vanishes. Indeed, the latter point is unphysical since it depends on the detailed regularization scheme [1].

An effective chiral Lagrangian provides the simplest approach to see how this structure arises. I build on the simplest linear sigma model involving the fields $\sigma$ and $\vec{\pi}$ interacting with a potential

$$
V=\lambda\left(\sigma^{2}+\vec{\pi}^{2}-v^{2}\right)^{2}-m \sigma
$$

Here $v$ represents the vacuum expectation value of $\sigma$ and $m$ is proportional to the average quark mass. The four meson coupling is denoted $\lambda$. The $\sigma$ field models the condensing combination $\bar{\psi} \psi$. The chiral transition occurs at $m=0$, where the order parameter $\langle\sigma\rangle$ jumps between $v$ and $-v$.

I now introduce isospin breaking into the picture. This will come from a non-vanishing mass difference between the down and up quarks. Without isospin symmetry, there is no reason for the neutral and charged pions to be degenerate. In particular, the breaking will allow the neutral pion to mix with isoscalar mesons. There are several candidates for this mixing, including the eta, the eta 


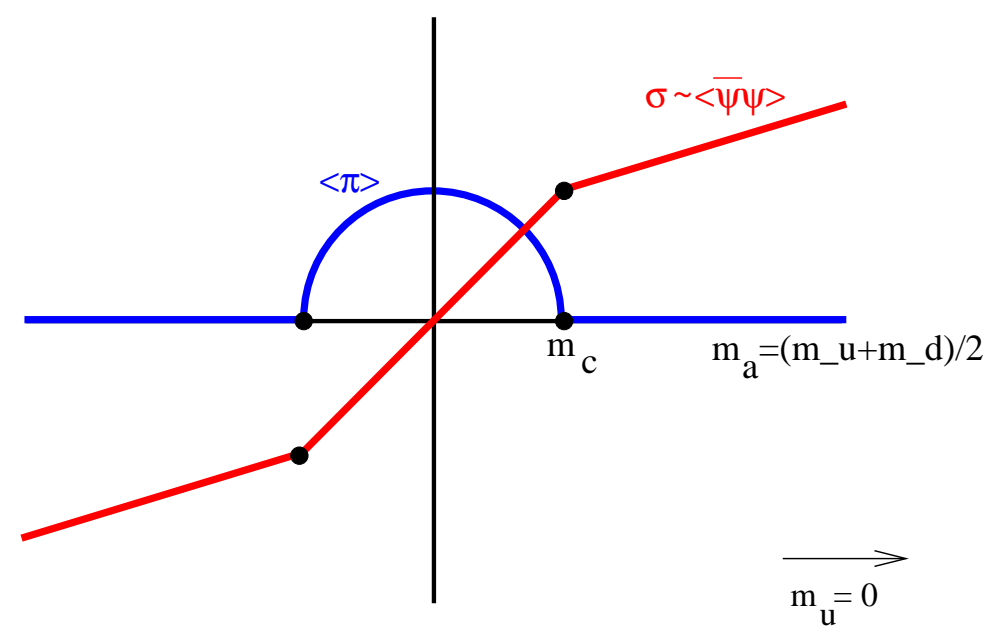

Figure 2: With a constant up-down quark mass difference, the jump in the chiral condensate splits into two second order transitions. The order parameter distinguishing the intermediate phase is the expectation value of the neutral pion field.

prime, and glueballs. As expected in quantum mechanics, this mixing will result in level repulsion. As the neutral pion is the lightest neutral state, the mixing inherently destabilizes the $\pi_{0}$ field.

Although several states are involved in this mixing, for pedagogy I simplify things and consider only a single effective isoscalar field $\phi$. Adding such a meson to the effective potential suggests one study

$$
V=\lambda\left(\sigma^{2}+\vec{\pi}^{2}-v^{2}\right)^{2}-m \sigma-\xi \pi_{0} \phi+m_{\phi}^{2} \phi^{2} / 2
$$

Here I have given the new field an initial mass $m_{\phi}$, and introduced the parameter $\xi$ to control the mixing between $\phi$ and $\pi_{0}$. The strength of this mixing should be proportional to the quark mass difference, $\xi \propto m_{d}-m_{u}$. Note that the sign of the mixing parameter $\xi$ is irrelevant as it can be changed by redefining $\phi$ as its negative.

Now consider minimizing the potential $V$ over fields. This gives the set of equations

$$
\begin{aligned}
& 0=\frac{\partial V}{\partial \sigma}=4 \lambda \sigma\left(\sigma^{2}+\pi_{0}^{2}-v^{2}\right)-m \\
& 0=\frac{\partial V}{\partial \pi_{0}}=4 \lambda \pi_{0}\left(\sigma^{2}+\pi_{0}^{2}-v^{2}\right)-\xi \phi \\
& 0=\frac{\partial V}{\partial \phi}=m_{\phi}^{2} \phi-\xi \pi_{0}
\end{aligned}
$$

The last of these allows elimination of $\phi$, resulting in

$$
\begin{aligned}
& \phi=\frac{\xi \pi_{0}}{m_{\phi}^{2}} \\
& \pi_{0}\left(\sigma^{2}+\pi_{0}^{2}-v^{2}-\frac{\xi^{2}}{4 \lambda m_{\phi}^{2}}\right)=0
\end{aligned}
$$


There are two generic possibilities for the value of $\pi_{0}$. At large $|m|$ the solution is CP conserving with

$$
\begin{aligned}
\pi_{0} & =0 \\
\sigma & = \pm v \sqrt{1+\frac{m}{4 \lambda \sigma v^{2}}} \sim \pm v .
\end{aligned}
$$

However at small $|m|$ there is a lower energy and CP violating solution

$$
\begin{aligned}
& \pi_{0}= \pm v \sqrt{1-\frac{m^{2} m_{\phi}^{4}}{v^{2} \xi^{4}}+\frac{\xi^{2}}{4 \lambda v^{2} m_{\phi}^{2}}} \\
& \sigma=\frac{m m_{\phi}^{2}}{\xi^{2}}
\end{aligned}
$$

This possibility of a CP violating solution was noted some time ago by Dashen [2]. The transition between the two behaviors occurs at the critical average mass

$$
m_{c}= \pm \frac{\xi^{2} v}{m_{\phi}^{2}} \sqrt{1+\frac{\xi^{2}}{4 \lambda v^{2} m_{\phi}^{2}}}
$$

This is of quadratic order in the mixing parameter, and thus is quadratic in the mass difference $m_{d}-m_{u}$. The transition is continuous, and the $Z_{2}$ symmetry of $\pi_{0} \leftrightarrow-\pi_{0}$ becomes spontaneously broken. Thus we expect the transition to be Ising like.

In these transitions the condensate difference $\left\langle\bar{\psi}_{u} \psi_{u}-\bar{\psi}_{d} \psi_{d}\right\rangle$ is automatically driven to a non-zero value by the explicit isospin breaking. Indeed, this difference should only display a rather weak dependence on average quark mass.

There are several minor variations on this argument that give rise to the same picture of a $\mathrm{CP}$ violating phase. In Ref. [3], the three flavor theory was studied containing a heavier strange quark; here mixing with the eta meson drives the phenomenon. A pure two flavor discussion [4] mixing the fields $\{\sigma, \vec{\pi}\}$ with the combination $\{\eta, \vec{\delta}\}$, where $\vec{\delta}$ is an isovector scalar field, showed a similar behavior.

As mentioned above, we expect this effect to be quadratic in the quark mass difference. The quark masses themselves should be small for or the chiral Lagrangian approach to be valid. Thus the critical mass should be even smaller than the individual quark masses themselves, i.e. $m_{c}=O\left(m_{q}^{2}\right)<<\left|m_{q}\right|$. Since the CP violation occurs when the average quark mass is less than the individual quark masses, the phenomenon requires quark masses of opposite sign. Although naively the signs of the quark masses can be changed by chiral rotations, their relative sign is protected by the anomaly. Furthermore, in this regime the sign of the fermion determinant is not always positive. This invalidates the Vafa-Witten [5] discussion suggesting that spontaneous CP violation cannot occur in the strong interactions.

At the critical average quark mass, the neutral pion is massless. This occurs at a point where neither of the quarks is itself massless. This shows that it is possible to have a massless meson without massless quarks. Indeed, immediately around the point where a quark mass vanishes, physics is analytic, as discussed by Di Vecchia and Veneziano [6]. Because of this smooth behavior, redefinitions of the underlying parameters result in an inherent ambiguity in defining where, say, 


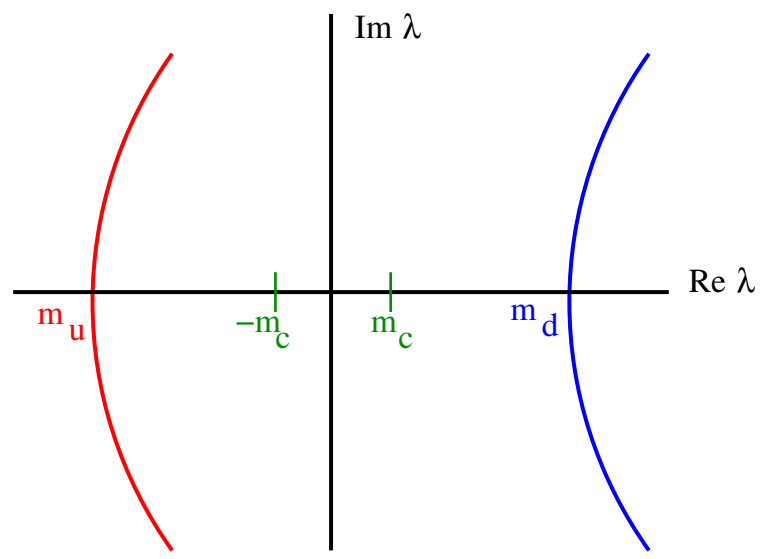

Figure 3: The eigenvalues of a Ginsparg-Wilson lattice fermion operator are constrained to lie on large circles. With a fixed up-down quark mass difference, the point where the neutral pion mass vanishes does not occur when either of these circles passes through the origin. Thus we can have long distance physics when none of the eigenvalues are small.

the up quark mass vanishes. As emphasized in Ref. [1], this means that contrary to popular lore, a vanishing up quark mass cannot be a fundamental solution to the strong $\mathrm{CP}$ problem.

While it might be interesting to study these transitions numerically in lattice gauge simulations, there are some technical obstacles that must be faced. As the fermion determinant is not positive when this phenomenon occurs, simulations will be afflicted with a sign problem. Furthermore, the mixing with isosinglet states occurs through disconnected quark diagrams, which therefore must be included.

With a lattice regulator that violates chiral symmetry, this $\mathrm{CP}$ violating phase can potentially survive even in the degenerate quark limit. This case represents the phase proposed some time ago by Aoki [7], and explored in the context of effective Lagrangians in Refs. [8, 9]. Depending on the sign of a certain parameter in the effective Lagrangian, the $\mathrm{CP}$ violating phase can either survive in the degenerate mass case or it could collapse into a single first order transition.

It is sometimes argued that long distance physics is controlled by low eigenvalues of the Dirac operator. The phenomenon discussed here is a counterexample to that concept. Consider a formulation in terms of a lattice Dirac operator satisfying the Ginsparg-Wilson [10] relation. An example of such an operator is provided by the overlap approach [11]. These operators force their eigenvalues onto large circles, the radii of which go to infinity as the continuum is approached. This is sketched in Fig. (3). These circles pass through the real axis at the corresponding bare quark masses. However, as discussed above, the transitions discussed in this paper do not occur when either quark mass vanishes, but at an average quark mass tuned to a value much less than the magnitude of either quark mass. At these transitions long distance physics manifests itself in the vanishing neutral pion mass, but this occurs away from any vanishingly small eigenvalues of the quark operators.

\section{Acknowledgements}

This manuscript has been authored under contract number DE-AC02-98CH10886 with the 
U.S. Department of Energy. Accordingly, the U.S. Government retains a non-exclusive, royaltyfree license to publish or reproduce the published form of this contribution, or allow others to do so, for U.S. Government purposes.

\section{References}

[1] M. Creutz, Phys. Rev. Lett. 92, 162003 (2004).

[2] R. F. Dashen, Phys. Rev. D 3, 1879 (1971).

[3] M. Creutz, Phys. Rev. Lett. 92, 201601 (2004) [arXiv:hep-lat/0312018].

[4] M. Creutz, Phys. Rev. D 52, 2951 (1995) [arXiv:hep-th/9505112].

[5] C. Vafa and E. Witten, Phys. Rev. Lett. 53, 535 (1984).

[6] P. Di Vecchia and G. Veneziano, Nucl. Phys. B 171, 253 (1980).

[7] S. Aoki, Phys. Lett. B 190, 140 (1987).

[8] M. Creutz, arXiv:hep-lat/9608024.

[9] S. R. Sharpe and R. L. . Singleton, Phys. Rev. D 58, 074501 (1998) [arXiv:hep-lat/9804028].

[10] P. H. Ginsparg and K. G. Wilson, Phys. Rev. D 25, 2649 (1982).

[11] H. Neuberger, Phys. Lett. B 417, 141 (1998) [arXiv:hep-lat/9707022]. 